\title{
Glucose intolerance, hyperinsulinaemia and cognitive function in a general population of elderly men
}

\author{
S. Kalmijn ${ }^{1,2,3}$, E. J.M. Feskens ${ }^{1}$, L.J. Launer ${ }^{1,2}$, T. Stijnen ${ }^{2}$, D. Kromhout ${ }^{1}$ \\ ${ }^{1}$ Department of Chronic Diseases and Environmental Epidemiology, National Institute of Public Health and Environmental \\ Protection, Bilthoven, The Netherlands \\ ${ }^{2}$ Department of Epidemiology and Biostatistics, Erasmus University Medical School, Rotterdam, The Netherlands \\ ${ }^{3}$ Netherlands Institute for Health Sciences, Rotterdam, The Netherlands
}

\begin{abstract}
Summary Cognitive impairment is highly prevalent among the elderly. Subjects with disturbed glucose metabolism may be at risk of impaired cognitive function, as these disturbances can influence cognition through atherosclerosis, thrombosis and hypertension. We therefore studied the cross-sectional association of cognitive function with hyperinsulinaemia, impaired glucose tolerance and diabetes mellitus in a population-based cohort of 462 men aged 69 to 89 years. Cognitive function was measured by the 30-point Mini-Mental State Examination. Results were expressed as the rate ratio (95\% confidence interval) of the number of erroneous answers given on the Mini-Mental State Examination by the index compared to the reference group. Compared to subjects with normal glucose tolerance, known diabetic patients had a rate ratio of $1.23(1.04-1.46)$, newly-diagnosed diabetic patients of $1.16(0.91-1.48)$
\end{abstract}

and subjects with impaired glucose tolerance of 1.18 (0.98-1.41), after adjustment for confounding due to age, occupation and cigarette smoking ( $p$ trend $=0.01$ ). Non-diabetic subjects in the highest compared to the lowest quartile of the area under the insulin curve had a rate ratio of $1.24(1.03-1.50)$, after adjustment for confounding ( $p$-trend $=0.02$ ). The results did not change appreciably when potentially mediating factors, including cardiovascular diseases and risk factors associated with the insulin resistance syndrome, were taken into account. These results suggest that diabetes, as well as impaired glucose tolerance and hyperinsulinaemia in non-diabetic subjects are associated with cognitive impairment. [Diabetologia (1995) 38: 1096-1102]

Key words Diabetes mellitus, glucose intolerance, insulin, cognition, aged, epidemiology.
Cognitive impairment is an important component of dementia, and a major determinant of the quality of life. It is important to identify risk factors for cognitive impairment that are potentially modifiable. There is now substantial evidence that in addition to diabetes mellitus, impaired glucose tolerance and hyperinsulinaemia are also associated with atheroscle-

Received: 16 December 1994 and in final revised form: 29 March 1995

Corresponding author: Dr. S. Kalmijn, Department of Chronic Diseases and Environmental Epidemiology, National Institute of Public Health, and Environmental Protection, P.O. Box 1, NL-3720 BA Bilthoven, The Netherlands

Abbreviations: NIDDM, Non-insulin-dependent diabetes mellitus; MMSE, Mini-Mental State Examination; CI, confidence interval; $R R$, rate ratio. rosis, thrombosis, and abnormal haemodynamic processes [1-4]. All these processes may have an aetiologic role in stroke and lacunar infarcts, which can subsequently lead to cognitive impairment and dementia. In addition, they are risk factors for white matter changes as seen on neuroimaging, which are associated with cognitive impairment [5].

Several clinical studies support the hypothesis that diabetes and hyperglycaemia may be related to reduced cognitive function [6-8]. However, little is known about the role of insulin in processes that may influence cognitive function. One study suggested, that among hypertensive patients, hyperinsulinaemia increased the risk of cognitive impairment [9]. To our knowledge, no community-based studies have examined the relationship of cognitive function to diabetes, impaired glucose tolerance and hyperin- 
sulinaemia. Therefore, we examined these relationships with data from the Zutphen Elderly Study [1]. In addition we also assessed the role of possible mediators of this relationship, including cardiovascular disease and risk factors associated with the insulin resistance syndrome.

\section{Subjects and methods}

Subjects. The Zutphen Elderly Study is a longitudinal study of risk factors for chronic diseases in men. It is a continuation of the Zutphen Study, which was initiated in 1960 as the Dutch contribution to the Seven Countries Study [10]. Zutphen is a small industrial town located in the eastern part of the Netherlands. Of the targeted 1266 men, 555 of whom were from the original cohort, born between 1900 and 1920, and 711 of whom were randomly selected from all other men in the same age range living in Zutphen, 939 participated in 1985. In the Spring of 1990,544 of 718 (76\%) surviving men were re-examined. Complete information on risk factors was available for 462 men in 1990, who formed the sample for the present cross-sectional analysis.

Examinations. An oral glucose tolerance test was performed according to World Health Organization (WHO) guidelines [11] in subjects without known diabetes. In the morning, after an overnight fast, a blood sample was obtained and then a glucose load of $75 \mathrm{~g}$ given. Blood samples were again taken after 1 and $2 \mathrm{~h}$. Fasting glucose concentration was determined for subjects known to have diabetes mellitus. Samples were collected in tubes with sodium fluoride. Plasma glucose was determined with the hexokinase method. Serum insulin was measured with a radioimmune assay (Pharmacia Diagnostics, Uppsala, Sweden). Within- and between-run coefficients of variation ranged from 6 to $7 \%$. The men were classified into three groups of glucose tolerance using the WHO criteria for diabetes and impaired glucose tolerance [11]. Diabetes was defined as a fasting glucose concentration over $7.8 \mathrm{mmol} / \mathrm{l}$ or 2 -h postload glucose concentration over $11.1 \mathrm{mmol} / \mathrm{l}$. Impaired glucose tolerance was defined as a fasting glucose concentration below $7.8 \mathrm{mmol} / 1$ and a $2-\mathrm{h}$ post-load concentration between 7.8 and $11.1 \mathrm{mmol} / \mathrm{l}$. Normal glucose tolerance was defined as both concentrations below $7.8 \mathrm{mmol} / \mathrm{l}$. In addition, a fourth group of known diabetic subjects was created on the basis of the medical interview, independent of their fasting glucose measure. This group included subjects who were treated with insulin, oral hypoglycaemic agents and diet. The area under the post-load glucose and the insulin curve was calculated with the trapezoidal rule ((fasting concentration*30 min) + (1$h$ concentration $* 60 \mathrm{~min})+(2-\mathrm{h}$ concentration $* 30 \mathrm{~min}))$. The area under the insulin curve correlated most strongly with 1-h post-load insulin concentration (1-h: $r=0.97,2-\mathrm{h}: r=0.71$, fasting: $r=0.71$ ).

Global cognitive function was tested with the Dutch version of the 30-point Mini-Mental State Examination (MMSE) [12]. The MMSE includes questions on orientation to time and place, registration, attention and calculation, recall, language and visual construction. Although this screening test was originally created for a clinical setting, it is extensively used in epidemiological studies, and has proven to be a reliable and valid indicator of cognitive impairment $[13,14]$. It was administered by two trained nurses in a controlled hospital setting. If the subject did not answer fewer than four individual items $(25 \%$ of the questions) these were rated as errors $(n=22)$ [15], except for items which could not be performed because of physi- cal disability, in which case a weighted total score was given $(n=10)$. If a subject did not answer four or more individual items the total MMSE score was considered missing $(n=5)$. We used a score of 26 or more as cut-off point for unimpaired cognitive function, since cognitive impairment is not very likely above this point. We used a score of 23 or less as an indicator of poor cognitive function, since this cut-off point has been shown to be indicative of dementia [14]. A score of either 24 or 25 was called borderline cognitive function.

Cardiovascular risk factors were obtained from a physical examination and a questionnaire, carried out by trained physicians. Body mass index (weight/height ${ }^{2}$ ) was calculated from height, measured to the nearest $\mathrm{mm}$ and body weight, recorded to the nearest $0.5 \mathrm{~kg}$ while the men were in underwear. Systolic and diastolic (fifth Korotkoff phase) blood pressure were measured with a random zero sphygmomanometer in duplicate on the right arm with the subject in supine position. The mean of the two blood pressure values was used in the analyses. Hypertension was defined as a systolic blood pressure greater than or equal to $160 \mathrm{mmHg}$ or a diastolic blood pressure greater than or equal to $95 \mathrm{mmHg}$ or the use of anti-hypertensive medication, regardless of blood pressure level [16].

Fasting and non-fasting blood was obtained to determine the concentrations of lipoproteins and haemostatic factors. Non-fasting serum HDL cholesterol was determined enzymatically after precipitation of apo lipoprotein B containing particles by dextran sulphate- $\mathrm{Mg}^{2}+[17]$ by the standardized Lipid Laboratory at the Department of Human Nutrition, Wageningen Agricultural University, The Netherlands. Fasting triglyceride was determined enzymatically by the same laboratory using a test kit (Boehringer Mannheim, Mannheim, Germany) [18]. Fibrinogen concentration was determined by the method of Clauss [19], in which the clotting time is measured in a solution of thrombin kept at constantly high concentration, added to diluted plasma. A standard curve is used to read the concentration of fibrinogen. Analyses were carried out at the Laboratory of the Department of Human Biology, University of Limburg, Maastricht, The Netherlands.

History of myocardial infarction, angina pectoris and intermittent claudication was obtained from the Dutch translation of a questionnaire developed at the London School of Hygiene and Tropical Medicine [20]. A standardized history of stroke, transient ischaemic attack and diabetes was also obtained. Medical records on all subjects, including ECGs, hospital discharge data and written information from general practitioners, were collected to verify diagnoses. Occupation was used as an indicator of intellectual capacity in this elderly Dutch population, that had had a reduced access to education. Lifetime occupation was obtained from a self-administered questionnaire, and coded from class one (professionals, managers and teachers) to class four (manual workers). Finally, smoking habits were assessed by the physician, on the basis of a standardized questionnaire.

\section{Statistical analysis}

Non-parametric tests (Kruskall-Wallis) were used to compare continuous skewed variables and chi-square tests to compare categorical variables. The number of erroneous answers on the MMSE, defined as 30 minus the MMSE score, followed a Poisson distribution. Therefore, multiple Poisson regression was used to estimate the rate ratio (RR) of the number of erroneous answers on the MMSE in the index vs the reference group. Thus, a rate ratio of 1.29 indicates that the number of erroneous answers on the MMSE in that group is on average 
Table 1. Selected characteristics of elderly men by level of cognitive function: the Zutphen Elderly Study, 1990

\begin{tabular}{|c|c|c|c|c|}
\hline \multirow[t]{2}{*}{ Characteristics } & \multicolumn{4}{|l|}{ Cognitive function } \\
\hline & $\begin{array}{l}\text { Poor } \\
\text { MMSE score } \leq 23 \\
(n=74)\end{array}$ & $\begin{array}{l}\text { Borderline } \\
\text { MMSE score 24-25 } \\
(n=76)\end{array}$ & $\begin{array}{l}\text { Unimpaired } \\
\text { MMSE score } \geq 26 \\
(n=312)\end{array}$ & $\begin{array}{l}\text { All } \\
(n=462)\end{array}$ \\
\hline Age (years) & $77.5 \pm 4.8^{\mathrm{a}}$ & $75.1 \pm 4.7$ & $74.4 \pm 4.3$ & $75.0 \pm 4.6$ \\
\hline Manual occupation (\%) & $36^{\mathrm{b}}$ & 26 & 21 & 25 \\
\hline Current cigarette smokers (\%) & 26 & 22 & 21 & 22 \\
\hline Triglycerides $(\mathrm{mmol} / \mathrm{l})$ & $1.38 \pm 0.70$ & $1.52 \pm 0.82$ & $1.44 \pm 0.76$ & $1.44 \pm 0.76$ \\
\hline Fibrinogen $(\mathrm{g} / \mathrm{l})$ & $3.75 \pm 0.34$ & $3.72 \pm 0.28$ & $3.68 \pm 0.33$ & $3.69 \pm 0.32$ \\
\hline \multicolumn{5}{|l|}{ Presence of $(\%)$ : } \\
\hline Stroke & 5 & 4 & 4 & 4 \\
\hline Transient ischaemic attack & 5 & 5 & 7 & 6 \\
\hline Myocardial infarction & 14 & 13 & 14 & 14 \\
\hline
\end{tabular}

Data given as mean $\pm \mathrm{SD} .{ }^{\mathrm{a}}$ Overall chi-square test, $p \leq 0.0001 ;{ }^{\mathrm{b}}$ overall chi-square test, $p \leq 0.001$

$29 \%$ higher than in the reference group. Because of extra Poisson variation, the standard errors of the estimated betas were computed using the robust (i. e. independent of the Poisson assumption) method described by Royall [21], and used to calculate the $95 \%$ confidence intervals (CI). The SAS computer program version 6.09 was used [22].

To examine the relationship between glucose tolerance and cognitive function, we categorized subjects into normal and impaired glucose tolerance, and newly-diagnosed and known diabetes, according to the WHO categories. For the analyses on insulin, subjects were categorized into quartiles of the area under the insulin curve, after excluding known and newly-diagnosed diabetic patients, because insulin concentrations usually decline when a subject develops diabetes, which makes insulin levels difficult to interpret. Age, occupation, and cigarette smoking (current: yes/no) were added to the model to adjust for potential confounding. We examined whether the association changed after adjusting for possible mediating factors: those associated with the insulin resistance syndrome (Body Mass Index, hypertension, HDL cholesterol, triglycerides, fibrinogen), and cardiovascular diseases, including stroke, transient ischaemic attack, myocardial infarction, angina pectoris and intermittent claudication. To examine whether glucose tolerance and insulin were independently related to cognitive function, we also adjusted for fasting insulin in the analysis on glucose tolerance, and for glucose tolerance in the analysis on hyperinsulinaemia. We also performed a joint analysis in which the 2-h glucose and the area under the insulin curve were both added to one model as continuous variables. As with the other analysis on hyperinsulinaemia, these analyses exclude known and newly-diagnosed subjects. Interactions between age and insulin or glucose concentrations, and between insulin and glucose concentrations, were investigated by including interaction terms in the models. All $p$-values are based on two-sided tests of statistical significance.

\section{Results}

The participants were aged 69 to 89 years, with a mean \pm SD age of $75 \pm 4.6$ years. The median MMSE score was 26 . Sixteen percent had a poor cognitive function (MMSE score $\leq 23$ ), and $31 \%$ had a MMSE score of 24 or 25 , indicating borderline cognitive performance. The subjects with the lowest scores were older and belonged more frequently to the manual occupation group (Table 1). Other characteristics were not significantly different across levels of cognitive function.

Cognitive function in diabetes mellitus and impaired glucose tolerance. Of the 37 known diabetic patients with complete information on risk factors, 8 were insulin treated. The subjects with impaired glucose tolerance were older than the other subjects (Table 2). Occupational history did not vary significantly by level of glucose tolerance. As expected, the mean area under the insulin curve was highest in the subjects with impaired glucose tolerance and lowest in the subjects with normal glucose tolerance. However, the correlation between the area under the insulin curve and 2-h glucose concentration in non-diabetic subjects was low $(r=0.19, p<0.001)$. Stroke was considerably more frequent in subjects with impaired glucose tolerance. The prevalence of poor cognitive function (MMSE score $\leq 23$ ) was highest in subjects with impaired glucose tolerance. After correction for confounding factors, including age, the number of erroneous answers on the MMSE increased with increasing glucose intolerance level $(p$ trend $=0.01$ ) (Table 2). Known diabetic subjects made $23 \%$ more errors in the MMSE, newly-diagnosed diabetic subjects $16 \%$, and subjects with impaired glucose tolerance $18 \%$, compared with subjects with normal glucose tolerance. The results did not change when potentially mediating factors such as stroke, other cardiovascular diseases and variables associated with the insulin resistance syndrome, were taken into account. There were no sig- 
Table 2. Selected characteristics and RRs (95\% confidence interval) of the number of incorrect responses to the MMSE by glucose tolerance status: the Zutphen Elderly Study, 1990

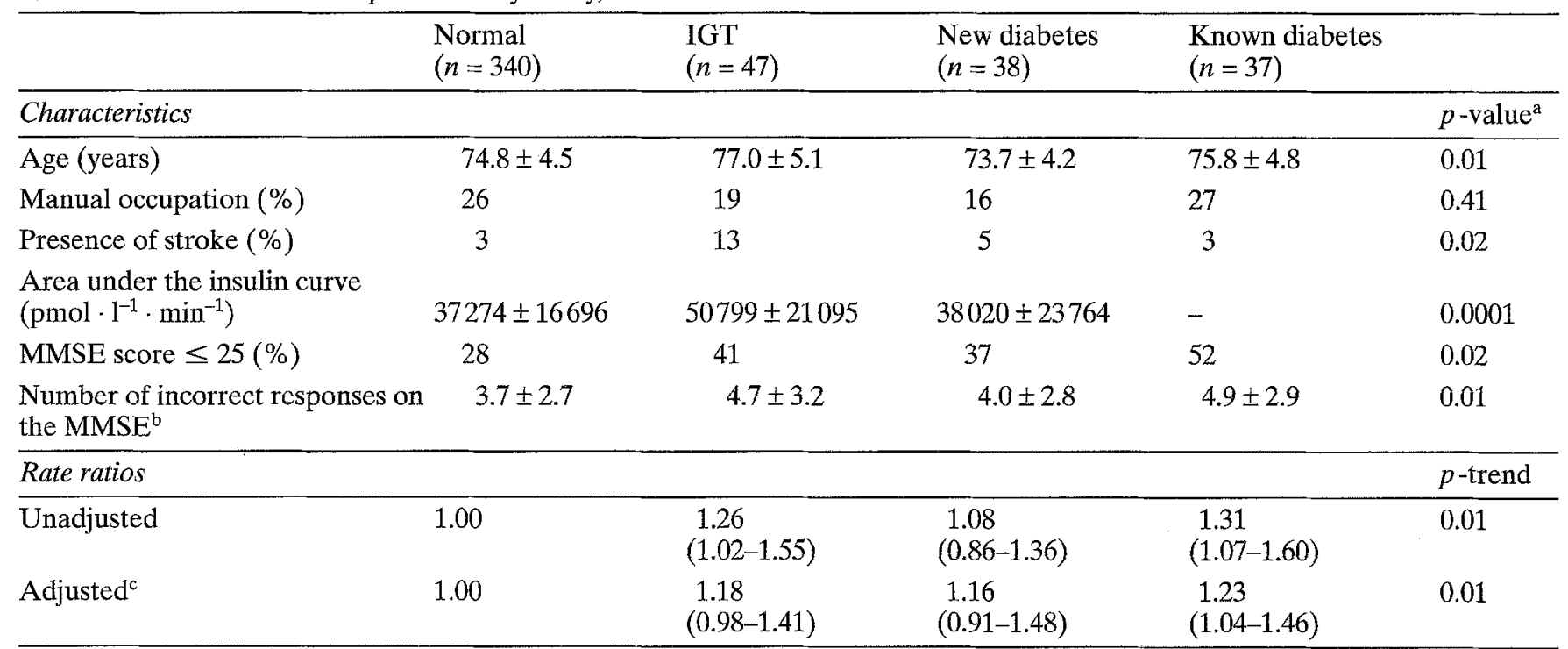

Data given as mean \pm SD. IGT, Impaired glucose tolerance $\quad$ ber of incorrect responses. ${ }^{c}$ Adjusted for age, occupation and

${ }^{a}$ Kruskall-Wallis test for continuous variables and chi-square

test for categorical variables. ${ }^{b}$ MMSE score $=30-$ mean num-

cigarette smoking

Table 3. Selected characteristics and RRs ( $95 \%$ confidence interval) of the number of incorrect responses to the MMSE by the level of area under the insulin curve in non-diabetic men: the Zutphen Elderly Study, 1990

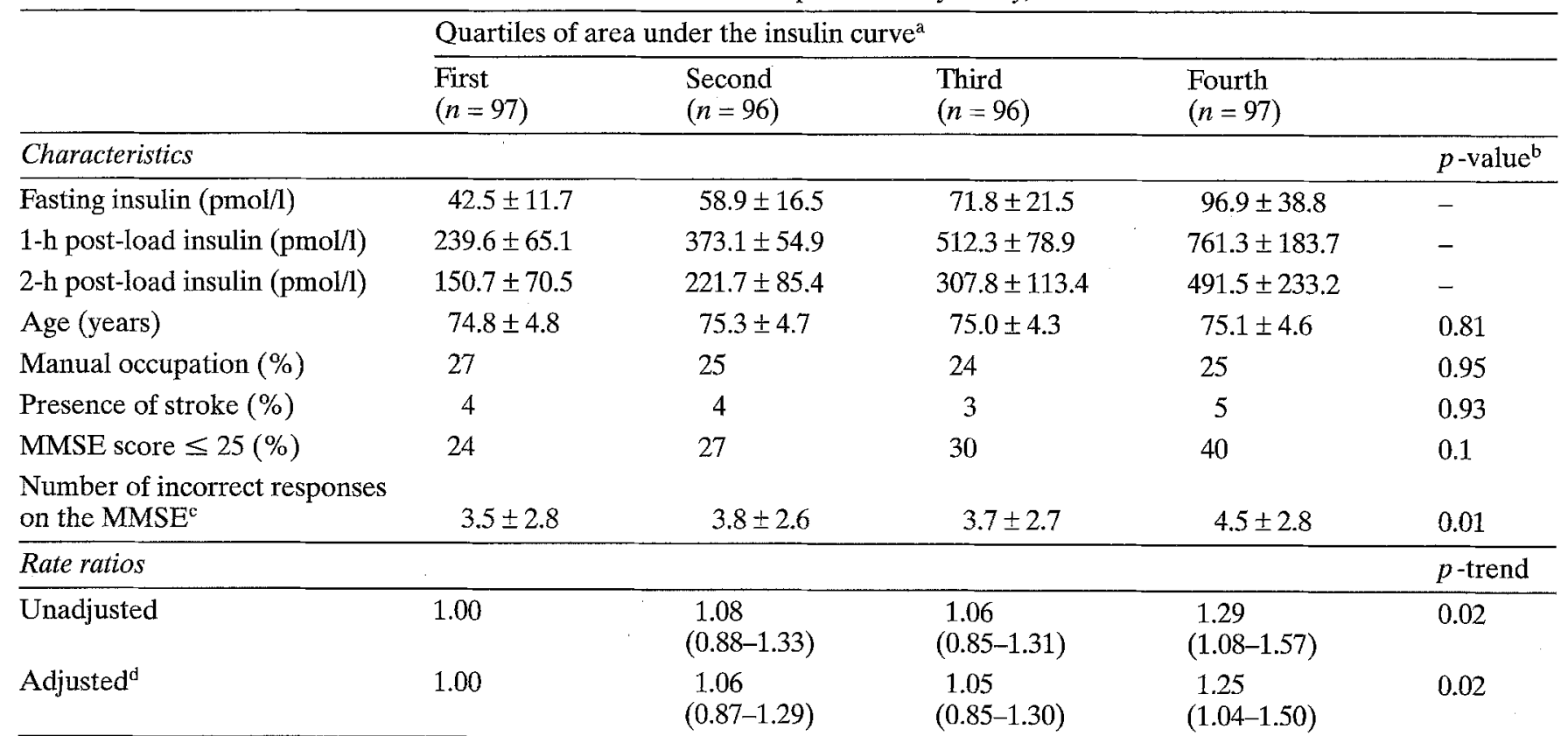

Data given as mean $\pm \mathrm{SD}$.

a First quartile: area under the insulin curve $\left(\mathrm{pmol} \cdot \mathrm{I}^{-1} \cdot \mathrm{min}^{-1}\right)$ $\leq 26347$, second quartile: $>26347$ to 35660 , third quartile: $>35660$ to 48065 , fourth quartile: $>48065$. ${ }^{\mathrm{b}}$ Kruskall-Wallis

nificant interactions between age and levels of glucose tolerance $(p>0.1)$.

When the analyses were confined to known and newly-diagnosed diabetic patients $(n=75)$ only, fasting glucose concentration was positively associated with the number of erroneous answers on the test for continuous variables and chi-square test for categorical variables. ${ }^{\mathrm{c}}$ MMSE score $=30-$ mean number of incorrect responses. ${ }^{d}$ Adjusted for age, occupation and cigarette smoking

MMSE. Median fasting glucose concentration of the subjects with diabetes was $8.0 \mathrm{mmol} / \mathrm{l}$ (10th centile: $6.3 \mathrm{mmol} / \mathrm{l}$, 90th centile: $12.4 \mathrm{mmol} / \mathrm{l})$. For each increase of $2 \mathrm{mmol} / 1$ subjects made $5 \%$ more errors in the MMSE, after adjustment for age and occupation ( $R R=1.05,95 \%$ CI:0.98-1.19). Eight of the 37 
known diabetic patients were using insulin. Their mean age at clinical diagnosis of diabetes was 61.5 years (range $40-84$ years). Exclusion of these eight patients did not change the results.

Cognitive function and hyperinsulinaemia. Among the 386 non-diabetic subjects age, occupation, and presence of stroke was comparable across the quartiles of the area under the insulin curve (Table 3 ). The percentage with poor cognitive function (MMSE score $\leq 23$ ) as well as the percentage with borderline cognitive function (MMSE score 24-25) increased from the lowest to the highest quartile of the area under the insulin curve. The mean MMSE score decreased from 26.5 in the lowest quartile to 25.5 in the highest quartile $(p=0.01)$. After adjustment for possible confounding effects of age, occupation and cigarette smoking, the subjects in the highest insulin quartile gave $25 \%$ more erroneous answers on the MMSE items compared with subjects in the lowest quartile (Table 3). Adjustment for potentially mediating factors, including cardiovascular diseases and factors associated with the insulin resistance syndrome, did not change the coefficient. Analyses pointed to a possible interaction between age and insulin quartiles $(p=0.09)$, suggesting that the association between hyperinsulinaemia and cognitive impairment was stronger in the older ( $>75$ years) than in the younger ( $\leq 75$ years) age group. Subjects older than 75 years in the highest insulin quartile made $45 \%$ more errors in the MMSE compared with those in the lowest quartile after adjustment for potential confounders (RR $=1.45,95 \%$ CI: $1.14-1.85$ ). Cognitive impairment was more strongly associated with the area under the insulin curve than with the fasting or post-load insulin concentrations. Comparing the highest to the lowest quartiles of hourly insulin measures, the rate ratio for 1 -h and 2 -h insulin concentrations was 1.16 (95\% CI:0.96-1.39) and 1.11 (95\% CI:0.92-1.33), respectively. The lowest rate ratio was found for the fasting insulin concentrations ( $\mathrm{RR}=1.08,95 \% \mathrm{CI}: 0.89-1.32)$.

The glucose tolerance and hyperinsulinaemia results were essentially the same when subjects with stroke were excluded from the analyses (results not shown). Additional adjustment for fasting insulin in the analysis on glucose tolerance did not change the results ( $p$-trend $=0.01$ ), neither did adjustment for glucose tolerance in the analyses on hyperinsulinaemia ( $p$-trend $=0.02$ ). In the joint analysis in non-diabetic subjects with the 2-h glucose and the area under the insulin curve as continuous measures, the rate ratio for 2 -h glucose was 1.01 per mmol/1 $(95 \%$ CI: $0.97-1.05)$ and 1.04 per $10 \mathrm{nmol} / 1^{-1} \cdot \min ^{-1}(95 \%$ CI: $1.00-1.07)$ for the area under the insulin curve. These rate ratios were not different from those obtained when each variable was entered separately in the model. There were no significant interactions be- tween levels of glucose tolerance and insulin quartiles $(p>0.1)$, indicating that the relationship between glucose tolerance and cognitive function did not vary across the quartiles of area under the insulin curve and vice versa.

\section{Discussion}

We found that non-diabetic subjects with impaired glucose tolerance and hyperinsulinaemia had impaired cognitive function as measured by the MMSE. Among diabetic patients cognitive function was even more impaired and decreased with increasing concentrations of fasting plasma glucose, which is an index of short-term glycaemic control. These results could not be explained by differences in age, occupation or smoking behaviour. Nor were they altered when subjects with stroke or diabetic patients using insulin were excluded. Joint analysis showed that hyperinsulinaemia was related to cognitive impairment, independent of glucose tolerance. Furthermore, diabetes and impaired glucose tolerance were related to cognitive impairment, independent of fasting insulin. Among non-diabetic subjects, the relationship of cognitive function to 2-h glucose and area under the insulin curve did not change when both glucose levels and area under the insulin curve were entered into the model.

There are a few methodological issues that should be taken into account when interpreting our results. First, as our study was cross-sectional, caution is needed regarding the direction of causality. Possibly, severe cognitive impairment leads to a deterioration of glycaemic control in diabetic patients. It is however not likely that cognitive impairment leads to glucose intolerance (i.e. diabetes or impaired glucose tolerance) or hyperinsulinaemia. Furthermore, selective non-response or survival may have affected the validity of this study. In another study, cognitive impairment and diabetes were shown to be more frequent in non-responders than responders [23]. It is also possible that subjects in whom diabetes and cognitive impairment are both present, have relatively higher mortality rates. If these selection biases apply to our study, it would probably result in an underestimation of the association between cognitive impairment and glucose intolerance or hyperinsulinaemia. We used the area under the insulin curve as an indicator of hyperinsulinaemia, rather than a single measurement of insulin. This area under the curve was a better predictor of cognitive function. This might be so because the within-subject variation of the area under the insulin curve is smaller than that of the hourly insulin measures [24]. Finally, serum insulin was measured with a conventional immunoassay, which is known to cross-react with proinsulin. Although proinsulin is known to be increased in diabe- 
tes and impaired glucose tolerance, it constitutes only a minor part of the total insulin measured [25] and is therefore probably not responsible for the observed association with cognitive function.

Our findings of impaired cognitive function in diabetic patients agree with earlier clinical studies showing first, that elderly patients with non-insulin-dependent diabetes mellitus (NIDDM) had poorer cognitive function compared with normal control subjects $[6-8,26]$, and secondly, that increasing levels of glycated haemoglobin in NIDDM patients were associated with poorer performance $[6,7]$. Meneilly et al. [26] showed that improvement of glycaemic control in patients with NIDDM increased selected aspects of cognition. In contrast, a longitudinal study found no significant relationship between NIDDM and cognitive impairment [27]. However, the subjects with NIDDM were young and had a high level of formal education. U'ren et al. [8] found that subjects not known to have diabetes, but who had raised glycated haemoglobin levels ( $>10 \%$ ), showed levels of cognitive performance intermediate between normal and diabetic subjects. This study is consistent with our finding that subjects with impaired glucose tolerance performed more poorly on the cognitive test compared to normoglycaemic subjects. Our finding of an association between hyperinsulinaemia and cognitive impairment is consistent with that of Kuusisto et al. [9], who found that hyperinsulinaemia identified a subgroup of hypertensive subjects with particularly impaired cognition.

We examined some potential explanatory mechanisms for the relation between glucose intolerance, hyperinsulinaemia and cognitive function. First, we investigated risk factors associated with the insulin resistance syndrome (hypertension, obesity, decreased HDL cholesterol, increased triglyceride concentrations and increased fibrinogen) [2-4] as mediating factors. The inclusion of these factors did not alter the results. Second, our adjustment for cardiovascular disease, as clinical manifestations of atherosclerosis, did not change the outcome. Moreover, exclusion of subjects with stroke did not alter the results. There is substantial evidence that glucose intolerance and hyperinsulinaemia are associated with atherosclerosis, thrombosis and hypertension [1, 4, 28], all of which may lead to cognitive impairment through cerebral infarcts or white matter disease [5]. Since atherosclerosis was not directly measured and we had no information on the severity of the cardiovascular disease, we cannot exclude this process as a mechanism explaining the association between glucose intolerance, hyperinsulinaemia and cognitive impairment. Furthermore, other mechanisms may be responsible for the poorer cognitive function in subjects with glucose intolerance and hyperinsulinaemia. The cognitive impairment of diabetic patients may be due to hypoglycaemia [29], increased free radical generation [30], or the same axonal damage as occurs in peripheral neuropathy [31]. Hyperinsulinaemia might affect cognitive function through vascular processes regulated by the sympathetic nervous system [32] or by influencing synaptic activity in the brain [33]. In future studies, information on the degree of atherosclerosis, the presence of cerebral infarcts or white matter disease, and the activity of the sympathetic nervous system, by measurement of plasma norepinephrine, would help to clarify the mechanisms through which insulin can affect cognitive function. Also important is to test aspects of cognitive function not measured by the MMSE, but that can be affected in diabetes, such as complex psychomotor function [34]. Further studies may also help to explain why dose-response was not strong and why the association between insulin and cognition varied with age.

In conclusion, cognitive function might not only be impaired in diabetic patients, but also in elderly subjects with impaired glucose tolerance and hyperinsulinaemia. In addition, poorer glycaemic control was associated with poorer cognitive function in diabetic patients. Clinicians should be alert to the possibility that patients with diabetes, impaired glucose tolerance or hyperinsulinaemia may be at risk for cognitive impairment.

Acknowledgements. This study was supported by grants from the Prevention Foundation, the Hague, the Netherlands and the National Institute on Aging, Bethesda, MD, USA. We thank the fieldwork team in Zutphen, especially E.B. Bosschieter, B.P.M. Bloemberg, and C. de Lezenne Coulander for data managements; M.A.J. de Ridder for statistical advice; I. Miedema for coding the cardiovascular disease data and A. Hofman for his critical review of the manuscript.

\section{References}

1. Feskens EJM, Kromhout D (1992) Glucose tolerance and the risk of cardiovascular diseases: the Zutphen Study. J Clin Epidemiol 45: 1327-1334

2. Reaven GM (1988) Banting lecture 1988. Role of insulin resistance in human disease. Diabetes 37: 1595-1607

3. DeFronzo RA (1992) Insulin resistance, hyperinsulinemia, and coronary artery disease: A complex metabolic web. J Cardiovasc Pharmacol 20[Suppl 11]:S1-S16

4. Juhan-Vague I, Thompson SG, Jespersen J (1993) Involvement of the haemostatic system in the insulin resistance syndrome. A study of 1500 patients with angina pectoris. Arterioscler Thromb 13: 1865-1873

5. Erkinjuntti T, Hachinski VC (1993) Rethinking vascular dementia. Cerebrovasc Dis 3: 3-23

6. Perlmuter LC, Hakami MK, Hodgson-Harrington C, Ginsberg J, Katz J, Singer DE, Nathan DM (1984) Decreased cognitive function in aging non-insulin-dependent diabetic patients. Am J Med 77: 1043-1048

7. Reaven GM, Thompson LW, Nahum D, Haskins E (1990) Relationships between hyperglycemia and cognitive function in older NIDDM patients. Diabetes Care 13: 16-21 
8. U'Ren RC, Riddle MC, Lezak MD, Bennington-Davis M (1990) The mental efficiency of the elderly person with type II diabetes mellitus. JAGS 38: 505-510

9. Kuusisto J, Koivisto K, Mykkänen L et al. (1993) Essential hypertension and cognitive function: the role of hyperinsulinemia. Hypertension 22: 771-779

10. Keys A, Aravanis C, Blackburn HW et al. (1967) Epidemiological studies related to coronary heart disease: characteristics of men aged 40-59 in seven countries. Acta Med Scand [Suppl]1-392

11. World Health Organization. Diabetes mellitus. Report of a WHO Study Group (1985) WHO technical report series 727. World Health Organization, Geneva

12. Folstein MF, Folstein SE, McHugh PR (1975) 'Mini-Mental State'. A practical method for grading the cognitive state of patients for the clinician. J Psychiatr Res 12: 189-198

13. Launer LJ (1992) Overview of incidence studies of dementia conducted in Europe. Neuroepidemiology 11[Suppl 1]:2-13

14. Siu AL (1991) Screening for dementia and investigating its causes. Ann Intern Med 115: 122-132

15. Fillenbaum GG, George LK, Blazer DG (1988) Scoring nonresponse on the Mini-Mental State Examination. Psychol Med 18: 1021-1025

16. Final Report of the Working Group on Risk and High Blood Pressure (1985) An epidemiological approach to describing risk associated with blood pressure levels. Hypertension 7: 641-651

17. Warnick GR, Benderson J, Albers JJ (1982) Dextran sulphate- $\mathrm{Mg}^{2+}$ precipitation procedure for quantitation of high-density-lipoprotein cholesterol. Clin Chem 28: 13791388

18. Sullivan DR, Kruijswijk Z, West CE, Kohlmeier M, Katan MB (1985) Determination of serum triglycerides by an enzymatic method not affected by free glycerol. Clin Chem 31: $1227-1228$

19. Clauss A (1957) Gerinnungsphysiologische Schnellmethode zur Bestimmung des Fibrinogens. Acta Hematol 17: $237-246$

20. Rose GA, Blackburn H (1968) Cardiovascular survey methods. World Health Organization, Geneva

21. Royall RM (1986) Model robust confidence intervals using maximum likelihood estimators. International Stat Rev 54: $221-226$

22. SAS Institute (1993) SAS Technical Report P-243 SAS/ STAT Software: The GENMOD Procedure, release 6.09, SAS Institute, Cary, NC
23. Launer LJ, Wind WA, Deeg DJH (1994) Nonresponse pattern and bias in a community-based cross-sectional study of cognitive functioning among the elderly. Am J Epidemiol 139: 803-812

24. Feskens EJM, Bowles CH, Kromhout D (1991) Intra- and interindividual variability of glucose tolerance in an elderly population. J Clin Epidemiol 44: 947-953

25. Reaven GM, Dhen Y-DI, Hollenbeck CB, Sheu WHH, Ostrega D, Polonsky KS (1993) Plasma insulin, C-peptide, and proinsulin concentrations in obese and nonobese individuals with varying degrees of glucose tolerance. J Clin Endocrinol Metab 76: 44-48

26. Meneilly GS, Cheung E, Tessier D, Yakura C, Tuokko H (1993) The effect of improved glycemic control on cognitive functions in the elderly patient with diabetes. J Gerontol 48:M117-M121

27. Robertson-Tchabo EA, Arenberg D, Tobin JD, Plotz JB (1986) A longitudinal study of cognitive performance in noninsulin dependent (type II) diabetic men. Exp Gerontol 21: 459-467

28. Fontbonne A, Charles MA, Thibult N et al. (1991) Hyperinsulinaemia as a predictor of coronary heart disease mortality in a healthy population: the Paris Prospective Study, 15-year follow-up. Diabetologia 34: 356-361

29. Kerr D, Diamond MP, Tamborlane WV, Kerr S, Sherwin RS (1993) Influence of counterregulatory hormones, independently of hypoglycaemia, on cognitive function, warning symptoms and glucose kinetics. Cli Sci 85: 197-202

30. Ceriello A, Quatraro A, Giugliano D (1993) Diabetes mellitus and hypertension: the possible role of hyperglycaemia through oxidative stress. Diabetologia 36: 265-266

31. Greene DA, Lattimer SA (1988) Pathogenesis and prevention of diabetic neuropathy. Diabetes Metab Rev 4: 201221

32. Rowe JW, Young JB, Minaker KL, Stevens AL, Pallotta J, Landsberg L (1981) Effect of insulin and glucose infusions on sympathetic nervous system activity in normal man. Diabetes 30: 219-225

33. Baskin DG, Figlewicz DP, Woods SC, Porte D, Dorsa DM (1987) Insulin in the brain. Ann Rev Physiol 49:335-347

34. Richardson JTE (1990) Cognitive function in diabetes mellitus. Neurosci Biobehav Rev 14: 385-388 